\title{
Selenomonas sputigena
}

National Cancer Institute

\section{Source}

National Cancer Institute. Selenomonas sputigena. NCI Thesaurus. Code C86734.

A species of strictly anaerobic, Gram negative, crescent shaped bacteria assigned to the phylum Firmicutes. This species is esterase positive, able to reduce nitrate and can produce acid from fructose, galactose, glucose, glycerol, lactose, maltose, melibiose, raffinose and sucrose. S. sputigena is found in human gingival spaces and is a cause of periodontal disease. 